\title{
Preference for familiar stimulation independent of fear of novelty ${ }^{\prime}$
}

\author{
ALICE B. SHELDON, George Washington University, \\ Washington, D. C. 20006
}

The hypothesis that stimulus familiarity generates positive motivation independent of aversive effects of novelty was investigated by offering animals three different degrees of familiarity, with novelty held constant. Preference was found to covary with degree of familiarity, regardless of sensory modality. The results tend to support J. McV. Hunt's proposal that perceptual familiarity has independent motivational effects.

J. McV. Hunt (1965) has proposed that animals display a positive motivation toward familiar stimuli which is independent of any aversive motive toward novelty. Some indications that perceptual familiarity elicits approach responses has been obtained by Hunt \& Uzgiris (1964), but the hypothesis of an independent motive generated by familiarity has not been directly tested. If there is a separate motivation toward familiar stimuli, it would be expected that the strength of animals' preference for a stimulus would vary with changes in the amount or intensity of familiarity alone, in the absence of changes in the amount of novel stimulation. The experiment reported here tested this prediction.

It has been shown that animals on first exposure to a strange environment elect to approach the more familiar of two comparable stimulus-objects (Sheldon, in press). This design offered a method for testing Hunt's hypothesis by offering the Ss three different degrees of sensory familiarity in the preferred object. The prediction under test was that preference would be strongest for maximal familiarity, weaker for intermediate familiarity, and least for minimal familiarity. Since these conditions did not involve concomitant increases in the level or amount of novelty, such a change in preference would tend to suggest the effects of an independent motive toward familiar stimulation.

\section{METHOD}

The experimental method has been fully reported in Sheldon (in press). Briefly, Ss were naive hooded rats approximately four months old, who had been made familiar with one stimulus object by having it placed in their cage for two weeks before test. The test consisted of placing the animal in a strange environment (a raised $Y$-runway), where he had the choice of entering a box containing his familiar stimulus or a box containing a comparable novel stimulus. The response measure was entry into a box, and only one trial was given per S. The stimulus which served as familiar for half the Ss was used as novel for the other half, to control irrelevant stimulus variables.

The experimental groups having different familiarity conditions are displayed graphically in Fig. 1.2

Maximal Familiarity

For this group (48 Ss), the familiar object was used as it came from the home cage, and was thus both visually and olfactorily familiar.

Intermediate Familiarity

For half of this group ( $24 \mathrm{Ss}$ ) the familiar stimulus was a clean duplicate of the home-cage object, and was thus only visually familiar. For the other $24 \mathrm{Ss}$, the familiar stimulus was the home-cage object from the complementary stimulus group, and was thus only olfactorily familiar (cage-odor of like-sex rats). These two intermediate conditions of reduced cues to familiarity are referred to as the visual and olfactory subgroups.

Minimal Familiarity or Competing Cues

For this group ( $24 \mathrm{Ss}$ ), the sensory cues to familiarity were opposed by offering them one object with only the familiar visual appearance vs another object having only olfactory familiarity. This represented a condition of minimal difference in familiaritv between the two stimuli.

\section{RESULTS}

Figure 2 shows the percentage of $S s$ who preferred the familiar stimulus under the three conditions of familiarity. Preference was strongest when the object offered both visual and olfactory familiarity, and declined about equally when either visual or olfactory familiarity was subtracted. There was no preference when familiar appearance and odor were opposed. The rank order by strength of preference correlated with the level of familiarity conditions $(r=1 ., \mathrm{df}=1, \mathrm{p}<.01)$.

$$
\text { DISCUSSION }
$$

The results show the predicted relation between the strength of preference, measured as the number of approach responses, and the overall amount of familiar stimulation, independent of the sensory modality used to present familiarity.

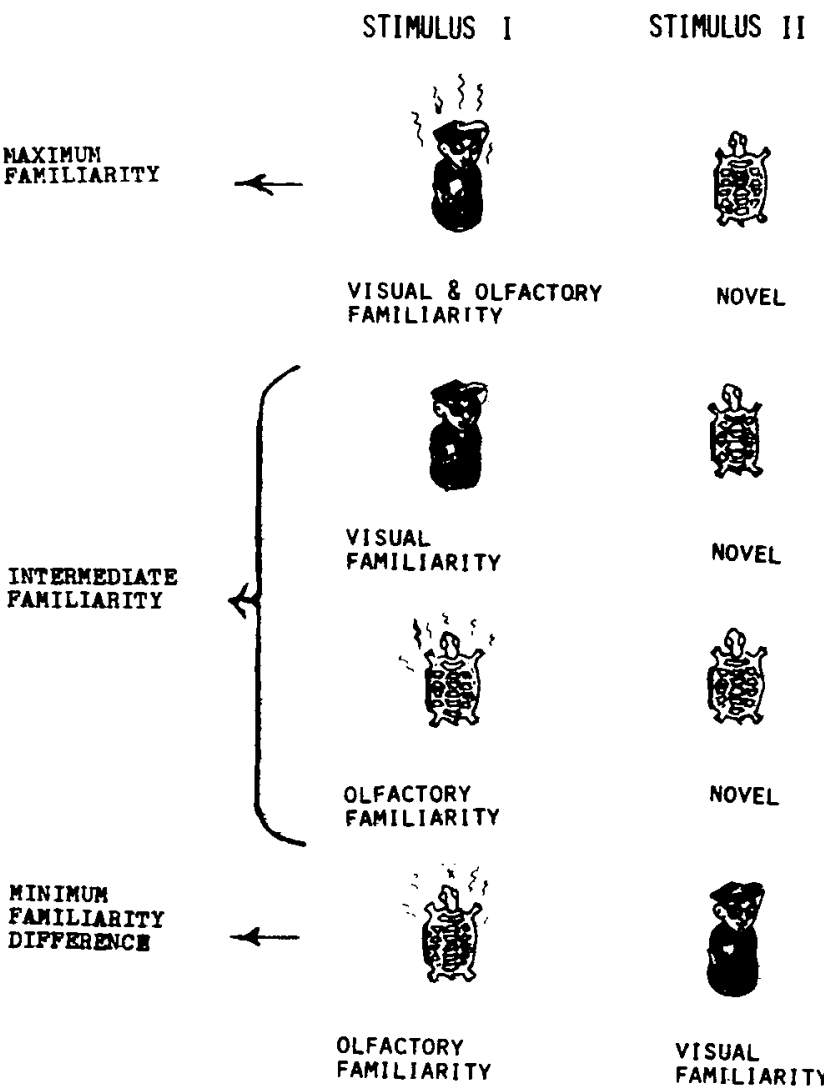

Fig. 1. The three different familiarity conditions, shown for a $S$ who had been made familiar with the glazed ceramic figure object. For the other half of the Ss, who had been made familiar with the metal locket object, the visual appearance of the stimulus pairs was the reverse of that shown here. 


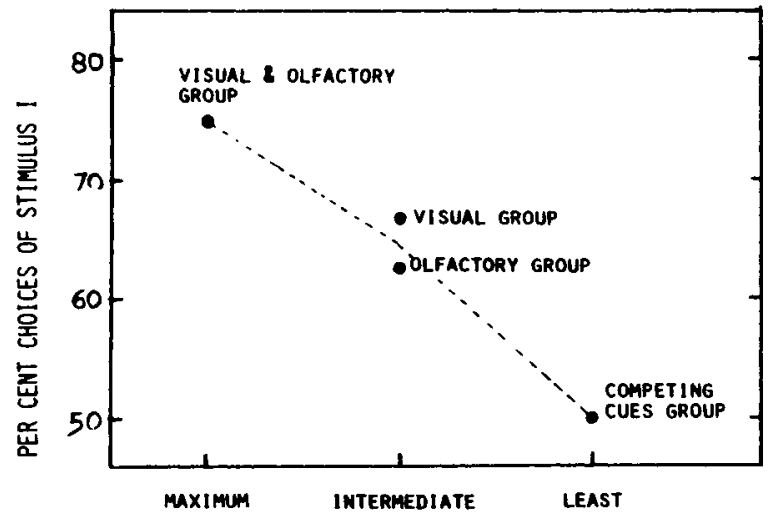

DIFFEREMTIAL FAMILIARITY OF STIMULUS I AND II

Fig. 2. The percentage of animals choosing the familiar stimulus at three different kevels of differentiol familiarity. (The results of the intermediate condition are shown separately for the subgroup having only visual familiarity and the subgroup having only olfactory familiarity.)

This orderly change in response cannot, it seems, be explained as the effects of a fear of novelty, because there was no commensurate increase in the amount or degree of novel stimulation in the intermediate or minimal familiarity conditions. In these conditions, the visually-familiar stimulus object had the same odor (of detergent) as the novel stimulus and the rest of the environment. Conversely, the olfactorilyfamiliar stimulus had the same appearance as the novel stimulus. Thus there was no "new" novelty added which could account for the declines in preference. Nor were there any differences in response measure or secondary reward aspects which might be appealed to. (See also Sheldon, 1968, for detailed control procedures.) With these alternative interpretations eliminated, it is most parsimonious to infer that the animals were responding to changes in the familiarity conditions alone, in the manner which would be expected if there were an independent positive motivation toward familiarity.

In sum, these data provide encouragement for Hunt's proposal that familiarity is not merely a zero condition of stimulation, but has behavioral effects deserving investigation.

\section{REFERENCES}

HUNT, J. McV. Intrinsic motivation and its role in psychological development. In D. Levine (Ed.), Nebraska symposium on motivation: 1965. Lincoln: University of Nebraska Press, 1965. Pp. 189-282.

HUNT, J. McV., \& UZGIRIS, I. C. Cathexis from recognitive familiarity. In P. R. Merrifield (Ed.), Experimental and factor-analytic measurement of personality. Kent, Ohio: Kent State University Press, 1968.

SHELDON, A. B. Preference for familiar or novel stimulation as a function of the novelty of the environment. Doctoral dissertation, George Washington University, Ann Arbor, Mich.: University Microfilms, 1968, No. 67-17, 303.

SHELDON, A. B. Preference for familiar versus novel stimuli as a function of the familiarity of the environment. Journal of Comparative \& Physiological Psychology, in press.

$$
\text { NOTES }
$$

1. This research was funded in part by Public Health Service Predoctoral Fellowship No. 10,907 and in part by grants from the National Science Foundation to Dr. Richard D. Walk. Acknowledgement is made to Drs. Walk, Lila Ghent, and Jacqueline Goodnow for their unstinting help.

2. For the Maximal group and the Visual subgroup, the single trial reported here was also the first trial of a serial test reported in Sheldon, in press. 